\title{
Predictions of the Interlaminar Tensile Failure of a Carbon/Epoxy Composite Laminate
}

\author{
A. Schiffer ${ }^{a}$ and V.L. Tagarielli ${ }^{b, *}$ \\ ${ }^{a}$ Department of Mechanical Engineering, KUSTAR, Abu Dhabi, UAE \\ ${ }^{\mathrm{b}}$ Department of Aeronautics, Imperial College London, SW7 2AZ, UK
}

\begin{abstract}
Finite element calculations are performed to model failure of a carbon/epoxy composite laminate loaded in tension in the through-thickness direction, and to predict the dependence of failure loads upon specimen size. The spatial variability of the inter-laminar strength is modelled by introducing different types of discrete random fields of material tensile strength. Fracture processes are modelled using the cohesive segment method, within the extended finite element framework of Abaqus Standard. Monte Carlo Simulation are conducted on different realisations of the random fields; the predicted responses are compared to previously published measurements and to reference FE simulations, in which the material strength is taken as uniform and equal to the measured average. The comparison shows that the modelling approach presented here provides more accurate predictions of the structural failure loads and their dependence on size, as well as capturing the failure modes observed in experiments.
\end{abstract}

Keywords: A. Laminate, B. Interfacial strength, C. Probabilistic methods, C. Finite element analysis (FEA)

Submitted to Composites Structures, February 2015

Revised article submitted in May 2015

\footnotetext{
*Corresponding author. E-mail v.tagarielli@imperial.ac.uk
} 


\section{INTRODUCTION}

Addressing the dependence of the strength of fibre-reinforced composites upon component size is an important aspect in the design of large naval and aeronautical composite constructions. Composites possess an irregular microstructure which results in spatial variations of material strength, giving rise to statistical size effects in their mechanical response.

A large body of measurements exists on the size dependence of the strength of fibre composites, and a comprehensive review is given in Wisnom [1]. Most of the experimental studies in the literature use the Weibull [2] distribution of strength to interpret the measurements. For the case of fibre-dominated failure modes, Jackson and Kellas [3] performed uniaxial tensile tests (along the fibre direction) on geometrically similar specimens made from carbon/epoxy composite and found that the strain at failure decreased by $\approx 20 \%$ when the specimens were scaled up by a factor of four, with the size effect characterised by a Weibull fit of modulus $m=24$. Other authors [4, 5] conducted scaled flexural tests on carbon-fibre/epoxy specimens and reported Weibull moduli in the range 13 to 25 .

Although several experimental studies showed good correlation with Weibull theory, this theory is based on a 'weakest link' assumption and cannot capture the progressive fracture mechanisms which have been reported by many authors (e.g. Rosen [6]). Better theories to describe the progressive failure processes of fibre composites have been developed, assuming that the composite can be idealised as a bundle of fibres which progressively fracture, see e.g. Wisnom [7] and Gurvich and Pipes [8].

Observation of microscopic damage in unidirectional carbon-fibre composites showed that small damaged clusters of fibres can be precursors to ultimate failure, as presented in Okabe and Takeda [9]. These authors performed Monte Carlo Simulations using a 3D micromechanical model for damage and found that traditional Weibull theory over-predicts the strength of the composite consequent to progressive fracture of fibres, as observed in the experiments. Numerical predictions based on a modified Weibull theory [10] provided good correlation with experimental results.

Fibre-composites with existing cracks exhibit a different type of size effect: Bazant et al. [11] conducted uniaxial tensile tests on notched specimens made from graphite/epoxy cross-ply 
and quasi-isotropic laminates and found a significant size effect on the nominal strength which was adequately represented by the asymptotic scaling model of Bazant [12].

When unidirectional fibre-composites are loaded in the through-thickness or transverse directions, failure is dominated by the fracture of the matrix. In this case Weibull theory captures experimental data well [1]. Statistical size effects are also more pronounced when failure is by matrix-dominated mechanisms. For example, O'Brian and Salpekar [13] investigated size effects in the transverse tensile response of carbon/epoxy laminates and reported a $30 \%$ decrease in the measured mean strength of specimens when their volume was increased by a factor of 60 . They also noted that ultimate failure was most likely caused by matrix microcracks and bonding defects between fibres and matrix. Other authors [14] performed scaled tests on curved unidirectional glass-fibre/epoxy beams in four-point bending to measure the size dependence of their interlaminar tensile strength. They showed that the strength can reduce by $44 \%$ as the dimensions are scaled up by a factor of four, and the corresponding Weibull modulus was $m=7.1$.

More recently, Tagarielli et al. [15] measured size effects in the response of a multidirectional composite laminate to direct through-thickness loading. The tensile interlaminar strength was characterised by a Weibull fit of modulus $m=13$, and to explore the dependence of strength upon volume, beams of different volume were tested in three-point bending, in order to load interlaminar interfaces by normal stresses, as sketched in Fig. 1a. The beams were observed to fail by propagation of interlaminar cracks. One of the objectives of the current investigation is to model the bending experiments of Tagarielli et al. [15].

The most common approach in mathematical modelling of the mechanical response of fibre composites is to use homogenised theories, which is convenient as the details of the complex and irregular microstructure are not directly considered. This approach is appropriate when the physical size of the material analysed (e.g. the volume of the finite element in an FE simulation) is larger than the representative volume element (RVE) used for the homogenisation; the RVE, in turn, needs to be sufficiently large in comparison to the relevant maximum length scale of the microstructure for the problem under investigation [16]. The effect of RVE size on the accuracy of the homogenisation is discussed in Drugan and Willis [17] and Kanit et al. [18], for the case of random linear elastic composites. 
If the geometry of the problem and the material microstructure do not allow a clear separation

\section{Uncertainty modelling and scaling of material strength}

\subsection{Quantification of uncertainty in material strength}

In this study a carbon-fibre/epoxy laminated composite of layup [0/45/0/-45 $]_{\mathrm{n}}$ and lamina thickness $a=0.3 \mathrm{~mm}$ was considered, as in Tagarielli et al. [15]. Direct interlaminar tensile tests were conducted on axisymmetric circular dogbone samples of gauge volume $V_{0}=$ 
$130 \mathrm{~mm}^{3}$, repeating the experiment more than 20 times. The specimens had a gauge section measuring $3.7 \mathrm{~mm}$ in diameter and $12 \mathrm{~mm}$ in length; they were manufactured by water-jet cutting circular cylinders of diameter $12.5 \mathrm{~mm}$ from a thick laminate; subsequently the cylinders were ground to produce the dogbone specimen geometry; the specimens were then mounted on a lathe and polished with strips of abrasive paper, down to a 4000 grit finish, to minimise the number of surface defects. The specimens were tested via specially design clamps which engaged by mechanical contact with the large ends of the dogbone specimens; such clamps were connected to a screw-driven testing machine by pin-joints, in order to avoid spurious bending. The strain was measured by miniature resistance strain gauges of geuge length $2 \mathrm{~mm}$ adhered to the lateral surface of the specimen, at the centre of the gauge portion.

In addition to the specimens described above, five additional tests were conducted on larger axisymmetric dogbone specimens, with gauge portion measuring $6 \mathrm{~mm}$ in diameter and length of $18 \mathrm{~mm}$. The volume of these larger specimens was approximately $510 \mathrm{~mm}^{3}$, as opposed to the $130 \mathrm{~mm}^{3}$ of the smaller dogbone specimens. The larger specimens were produced via an identical manufacturing route as described above.

Both large and small specimens failed by macroscopically brittle interlaminar fracture, without any notable permanent deformation. The measured cumulative probability of survivability $P_{s}$ for the smaller specimens (of volume $130 \mathrm{~mm}^{3}$ ) fitted adequately a Weibull distribution

$$
P_{s}\left(\sigma, m, \sigma_{0}\right)=\exp \left[-\left(\frac{\sigma}{\sigma_{0}}\right)^{m}\right]
$$

with reference stress $\sigma_{0}=75 \mathrm{MPa}$ and Weibull modulus $m=13$. Note that the average strength measured in these tests was $\bar{\sigma}=70 \mathrm{MPa}$. The larger specimens had an average strength $\bar{\sigma}=66 \mathrm{MPa}$. The scatter in the measurements was of approximately $20 \%$ over the limited number of repetitions performed.

If the probability of survivability $P_{s}$ of a material specimen of volume $V_{0}$ follows a Weibull distribution (1), the $P_{s}$ of a specimen of different volume $V \neq V_{0}$ subjected to uniform stress $\sigma$ is then given by 
By substituting in eq. (2) $V_{0}=130 \mathrm{~mm}^{2}$ and $V=510 \mathrm{~mm}^{2}$, this equation predicts an average strength of $64 \mathrm{MPa}$ for the larger specimens, not far from the measured value of $66 \mathrm{MPa}$.

For non-uniform stress conditions, eq. (2) can be extended to

$$
P_{s}\left(\sigma, V, m, \sigma_{0}, V_{0}\right)=\exp \left[-\frac{1}{V_{0}} \int_{V}\left(\frac{\sigma(\underline{x})}{\sigma_{0}}\right)^{m} d V\right]
$$

where the stress distribution $\sigma(\underline{x})$ is integrated over the sample volume to account for the fact that material points subject to higher stresses make larger contributions to the probability of failure. Equation (3) assumes that only one component of the stress tensor contributes to the probability of failure of the component or specimen.

The experimental evidence suggests that the the material is affected by a statistical size effect, due to the stochastic distribution of the interlaminar tensile strength at the interface between adjacent plies, where fracture initiates and progresses [15]; this distribution is described with sufficient accuracy by a Weibull distribution, however the measurements might have been fitted with similar probability distribution with similar accuracy; we choose to adopt the Weibull distribution for uniformity with the published literature on this topic. We assume that the measured strength is not influenced by the presence of the shear components of the interlaminar stress state.

\subsection{Scaling models for failure of beams in three-point bending}

In this section, we employ eq.(3) to construct two simple theoretical scaling models for the strength of beams in three-point bending; these rely on the following assumptions: (i) beam theory is valid for the structures investigated, (ii) interlaminar shear stresses and free edge effects are small and do not contribute to the local probability of failure, (iii) failure is always initiated in the lower half of the beam, subject to tensile normal stresses, and (iv) failure proceeds unstably from the initiation point. In view of these assumptions, the theoretical models can only predict the onset of failure of slender beams and are used here as a reference for comparison to experiments and numerical predictions. 
For a linear-elastic, prismatic beam of depth $h$ and width $b$, simply supported across the span

$$
\sigma_{x x}(x, y, z)=\frac{6 P z}{b h^{3}}\left(\frac{L}{2}-x\right)
$$

in the coordinate system $(x, y, z)$, see Fig. 1a. Substituting the latter equation into eq.(3) and integrating over the lower half of the beam gives

$$
\frac{\sigma_{f}}{\sigma_{0}}=\left[-2 \ln \left(P_{s}\right)(m+1)^{2}\right]^{\frac{1}{m}}\left(\frac{V}{V_{0}}\right)^{-\frac{1}{m}},
$$

where $V$ is the beam volume, $\sigma_{f}=3 P_{f} L /\left(2 b h^{2}\right)$ is the maximum bending stress at failure (denoted in the following as apparent material strength) and $P_{f}$ is the failure load. As $P_{s}$ varies from 0 to 1 , eq. (5) predicts the corresponding expected value of the apparent material strength. Equation (5) is independent of the beam's aspect ratio $h / L$.

Equation (5) is typically used to predict the size effect associated with brittle failure of linearelastic beams in three-point bending. For the material and the tests considered here, experimental evidence [15] suggests that initiation of fracture is in the resin rich interfaces between adjacent laminas (see Fig. 1b); cracks may jump across adjacent laminae during their unstable propagation. Assuming that fracture processes exclusively initiate at such interfaces, the Weibull probability of survivability can be formulated in terms of the interface area

$$
P_{s}\left(\sigma, A, m, \sigma_{0}, A_{0}\right)=\exp \left[-\frac{A}{A_{0}}\left(\frac{\sigma}{\sigma_{0}}\right)^{m}\right]
$$

for uniformly stressed samples and

$$
P_{s}\left(\sigma, A, m, \sigma_{0}, A_{0}\right)=\exp \left[-\frac{1}{A_{0}} \int_{A}\left(\frac{\sigma(\underline{x})}{\sigma_{0}}\right)^{m} d A\right]
$$

in the presence of stress gradients; in eqns. (6) and (7), $A_{0}$ and $A$ are the total area of interfaces within the reference specimen (on which the Weibull distribution is calibrated) and within the actual sample, respectively.

Consider three-point bending of a composite laminate, comprising of a stack of $N_{L}$ laminae of thickness $a$, with equidistant interfaces parallel to the $y$ - $z$ plane, as sketched in Fig.1a, and as in the tests [15]. Re-write eq.(7) as 
with $A_{i}=b h$ the area of a single interface and $A=b h L / a$ the total area of interfaces contained within the entire specimen volume. Making use of the stress distribution (4) and integrating (8) over the portion of fracture surface subject to tensile stresses $(z>0)$ gives

$$
\frac{\sigma_{f}}{\sigma_{0}}=f\left(P_{s}, m, N_{f}\right)\left(\frac{A_{i}}{A_{0}}\right)^{-\frac{1}{m}} .
$$

for the apparent material strength $\sigma_{f}$. The apparent strength now depends on the number of interfaces $N_{f}=N_{L}-1$ and scales with $A_{i}$ according to a power-law exponent $-1 / m$. In the problem under investigation the cross-sectional area of the beam and its volume are proportional; therefore, the pairs of parameters $m=13$ and $\sigma_{0}=75 \mathrm{MPa}$ to be used in (5) and (9) are identical. The remaining parameters, $V_{0}=130 \mathrm{~mm}^{3}$ and $A_{0}=430 \mathrm{~mm}^{2}$, are the total volume and the total interface area of the gauge sections of the tensile specimens tested by Tagarielli et al. [15], respectively.

Figure 2 presents apparent strength predictions as functions of beam volume $V$ (Fig. 2a) and total interface area $A_{f}=N_{f} A_{i}$ (Fig 2b), both obtained via eq. (9) with $P_{s}=0.01$ and for different values of $N_{f}$. Figure 2a shows that for a given beam volume, an increase in the number of interfaces $N_{f}$ leads to a decrease in apparent strength, with the magnitude of the size effect preserved (i.e., the curves in Fig. $2 \mathrm{a}$ have identical slope). In Fig. $2 b$ the same predictions are plotted as a function of the total interface area $A_{f}$; clearly the sensitivity of the predictions to $N_{f}$ is very low for $N_{f}>29$.

\section{Numerical simulations}

We proceed to present the details of the numerical simulations conducted. We use the Monte Carlo Simulation method, conducting repeated FE simulations on different realisations of random fields of material properties, and analysing the outputs. Each simulation is conducted using ABAQUS/Standard. While the elastic response of the composite laminae is modelled as homogeneous, the failure parameters of the interfaces are taken as spatially variable according 
to appropriate probability distributions. For the purpose of comparison, deterministic

\subsection{Reference FE simulations}

We model prismatic beams of depth $h=3 \mathrm{~mm}$ and span $L=25 \mathrm{~mm}$, loaded in three-point bending via rigid cylindrical rollers of radius $R=6 \mathrm{~mm}$, in order to mimic the boundary conditions in the experiments (see Fig. 1a). The beam width $b$ was either 3,15 or $53 \mathrm{~mm}$. Contact between beam and rollers was modelled by a surface-to-surface algorithm with frictionless tangential behaviour. The supports were considered to be fixed with all degrees of freedom constrained to zero; loading was applied by imposing on the top roller a linearly ramped displacement $u_{z}=1.2 \mathrm{~mm}$ (this values was sufficient to fail the beams). The beam was meshed using fully integrated 8-noded hexahedral brick elements (C3D8 in ABAQUS) and the mesh size was chosen to be $l_{x}=0.3 \mathrm{~mm}$ in the $x$-direction, giving one element per lamina. To accurately model the sliding contact at the supports, the mesh was refined to an element length of $0.15 \mathrm{~mm}$ along the $x$-direction, in proximity of the contact points. In the $z$ direction a mesh size of $l_{z}=0.2 \mathrm{~mm}$ was chosen, and in the $y$-direction, the element length $l_{y}$ was set to $0.6,1.5$ or $2.12 \mathrm{~mm}$ for beams of width $b=3 \mathrm{~mm}, 15 \mathrm{~mm}$ or $53 \mathrm{~mm}$, respectively. Reference FE simulations were also conducted with a refined mesh (in all directions) and no significant differences in the results were detected, suggesting that the chosen discretization is adequate to achieve mesh independent results.

Two preliminary sets of simulations were conducted: in the first set, the material was modelled as isotropic linear elastic, with properties $E=7 \mathrm{GPa}$ and Poisson's ratio $v=0.2$ taken from the measurements (Tagarielli et al. [15]); in the second set, the material was modelled as a linear elastic, layered solid, with each layer representing a transversely isotropic lamina. The comparison showed that the elastic bending response was unaffected by the anisotropy of the material and that the shear components of the interlaminar stress were one order of magnitude lower than the normal interlaminar stress. We thereby assume that failure of the interfaces is driven primarily by normal stresses and in the following only the results obtained with the isotropic material properties are presented. 
The onset and evolution of failure was modelled using the cohesive segment method within

No pre-existing cracks were considered in our models; the properties of the XFEM enriched domain were set in ABAQUS such that cracks could nucleate and extend during the analysis once the maximum tensile principal stress in an element reached a critical threshold $\sigma_{\mathrm{f} 0}$; the newly inserted or extended crack is always orthogonal to the direction of the largest principal tensile stress. The separation of crack faces, as determined by the phantom node method [24], was controlled by a linear cohesive softening response (this is conceptually similar to the more typical interface-based cohesive behaviour). For a combination of normal and shear separations, $\delta_{n}, \delta_{s}$ and $\delta_{t}$, respectively, ABAQUS uses an effective separation defined as

$$
\delta_{m}=\sqrt{\left\langle\delta_{n}\right\rangle+\delta_{s}+\delta_{t}} .
$$

The rate of softening was assumed to be mode-independent and set by specifying the fracture energy $G_{f}$, defined as the area under the cohesive traction-separation curve. In ABAQUS, the damage variable $D$, ranging from 0 to 1 , describes to which degree the cohesive stiffness at the crack tip is degraded. The normal and shear stress tractions of a cracked element $t_{\mathrm{n}}, t_{\mathrm{s}}$ and $t_{\mathrm{t}}$, respectively, are affected by $D$ according to

$$
\begin{aligned}
& t_{n}=\left\{\begin{array}{cc}
(1-D) T_{n} & \text { for } T_{n} \geq 0 \\
T_{n} & \text { otherwise }
\end{array}\right. \\
& t_{s}=(1-D) T_{s} \\
& t_{t}=(1-D) T_{t}
\end{aligned}
$$

where $T_{\mathrm{n}}, T_{\mathrm{s}}$ and $T_{\mathrm{t}}$ represent the corresponding elastic stress predictions for the current configuration without damage. The cohesive failure model introduces in the simulations a characteristic material length scale

$$
\delta_{f}=\frac{E G_{f}}{\sigma_{\mathrm{f} 0}^{2}},
$$

associated with the size of the fracture process zone. 
In absence of more accurate measurements, the fracture energy was set at $G_{f}=300 \mathrm{Nm}^{-1}$, a value typically deduced from analysis of double cantilever tests on similar carbon/epoxy laminates, loaded in Mode I. Simulations were also performed with much higher values (up to $G_{f}=12,000 \mathrm{Nm}^{-1}$ ) in order to explore the sensitivity of the predictions to this parameter.

To aid convergence of the FE calculations, local damping was introduced using the viscous regularization technique. The viscosity was chosen such that the energy dissipated by damping was smaller than $1 \%$ of the total strain energy; a viscosity parameter of $\eta=10^{-4}$ was sufficiently small to satisfy this criterion, yet large enough to enable convergence of the calculations during the softening phase.

\subsection{Monte Carlo Simulations (MCS)}

The MCS presented in this study were based on the reference model described in Section 3.1, and repeated simulations were performed on different realisations of random fields of material strength in order to obtain the average strength and the scatter in the predicted response. In each simulation we use a random number generator in order to prescribe an initial field of fracture properties onto the FE discretisation. We assume a randomly varying failure initiation stress $\sigma_{\mathrm{f} 0}$ and a uniform fracture energy $G_{f}$ (typically, $G_{f}=300 \mathrm{Nm}^{-1}$ ), and implement this over all finite elements $V_{e}$ of the discretisation $\left(V=\cup_{e=1}^{N_{e}} V_{e}\right)$.

We introduce the notion of a statistical discretisation $V=\cup_{i=1}^{N_{i}} V_{i}$, consisting of $N_{i}$ stochastic volume elements (SVE), each of volume $V_{i} \geq V_{e}$. Such stochastic mesh allows implementing a discrete random field (RF) of failure stress $\sigma_{\mathrm{f} 0}$ over the solution domain. We assume that cracks exclusively nucleate at interlaminar interfaces and we therefore use the probability of survivability (6) in order to generate random fields of the property $\sigma_{\mathrm{f} 0}$ across the stochastic mesh by generating Weibull-distributed numbers for $\sigma_{\mathrm{f} 0}$ according to eq. (6), where $A$ is taken as the interface area contained in each SVE. We employ two different approaches in choosing the random field, as described below. 
(i) Homogeneous interfaces:

In this approach we consider each interface as a single homogenous defect, with uniform failure stress (i.e., independent of the $y$ - and $z$-directions). This is enforced by choosing the RF1 representation of random strength (see Fig. 3a), where the SVE size was chosen to be $0.3 \mathrm{~mm}$ in the $x$-direction in order to coincide with the lamina thickness and with the FE mesh (i.e. one interface is fully enclosed in one SVE). The corresponding SVE model developed in ABAQUS is shown in Fig. 3a (RF1, top figure), illustrating both the FE discretisation of the beam (black wireframe) and the statistical mesh (coloured patches).

\section{(ii) Heterogeneous interfaces:}

Here, the statistical mesh is refined to allow for variation of material strength over the plane of each of the interfaces, as illustrated in Fig. 3a with RF4 (bottom figure). Observation of the microstructure in [15] (Fig. 1b) does not provide enough detail to identify the length scale over which the material strength varies across an interface; therefore we consider four different types of random fields, denoted here as RF2, RF3, RF4 and RF5, respectively, where increasing numbers denote decreasing SVE size in the statistical mesh (see Fig. 3b). With exception of RF5, the FE mesh size is identical in all the random fields considered here.

Table 1 provides a summary of all the MCS performed in this study, and reports the finite element and statistical mesh sizes (i.e. SVE size) used in these simulations. In each simulation, a MATLAB script was used to edit automatically the input file of the deterministic simulations, in order to assign different traction-separation laws to each SVE and to all finite elements contained within it, in accordance to the chosen RF and based on the area of interfaces contained in each SVE, through eq. (6). Each simulation was repeated $N_{\text {sim }}$ times in order to obtain the population of the ensemble response.

In this study, $N_{\text {sim }}$ was chosen based on preliminary MCS performed with RF4 on a beam of width $h=3 \mathrm{~mm}$. In these simulations the predicted standard deviation and average strength was calculated from the response population after 10 and 20 realisations, respectively. The 
comparsion showed only minor differences in the predicted response (less than 7\%) and therefore we chose $N_{\text {sim }}=10$ in all MCS presented here.

\section{RESULTS AND DISCUSSION}

\subsection{Reference simulations}

We now proceed to present results obtained from the deterministic reference simulations. Figure 4a illustrates the failed configuration of a beam with $L=25 \mathrm{~mm}, h=3 \mathrm{~mm}, b=3 \mathrm{~mm}$ and spatially uniform fracture parameters $\left(\sigma_{\mathrm{f} 0}=70 \mathrm{MPa}, G_{f}=300 \mathrm{Nm}^{-1}\right)$. Note that the fracture stress $\sigma_{\mathrm{f} 0}$ was chosen to equal the average interlaminar strength measured in tension for this laminate, see Section 2.1. It can be seen that the crack originates at mid-span and at the centre of the lower face, propagating towards the top face (the red circles indicate FE nodes adjacent to the mid-plane). In proximity of the contact region the stress field induced by the loading roller forces the crack to deviate from its original straight path.

The crack front contours in the $y-z$ plane at mid-span are presented in Fig. $4 \mathrm{~b}$ for five selected instants following the onset of failure, while the applied bending stress $\sigma_{x}=3 P L /\left(2 b h^{2}\right)$ is given in Fig. 4c as a function of mid-span displacement; markers in Fig. 4c correspond to the five crack front contours in Fig. $4 \mathrm{~b}$. It can be seen from Fig. $4 \mathrm{~b}$ that a crack originates at the centre of the bottom face, extends towards the edges and continues its propagation towards the top face, with the crack front at the edges slightly trailing behind. It is clear from Fig. 4c that the crack propagation was unstable, as the peak in loading was reached at the onset of cracking.

\subsection{Monte Carlo simulations}

In this section, results of the MCS are presented and numerical predictions are compared to analytical models and experiments. 


\subsubsection{Sensitivity of the response to the random field imposed}

Figure 5a shows the final, cracked configuration of two beams $(b=3 \mathrm{~mm})$, predicted by two realisations of RF1 (denoted here as $\mathrm{S} 1$ and $\mathrm{S} 2$ ), with spatially varying fracture stress, $\sigma_{\mathrm{f} 0}$, and constant fracture energy, $G_{f}=300 \mathrm{Nm}^{-1}$. It can be seen that in both cases the crack did not originate at mid-span (indicated by the red circles), due to the variations of strength along the $x$-direction (see Fig. 3a). The corresponding crack front contours are presented in Fig. 5b for five selected instants. In both cases, the crack initiated at the bottom face halfway between the bottom edges and propagated towards the top face, with the crack front at the edges slightly trailing behind the central portion, similar to what observed in Fig. 4b. The crack again propagated unstably in both cases, as it can be seen from Fig. 5c where the applied bending stress is plotted as a function of the transverse displacement, clearly showing that for both realisations $\mathrm{S} 1$ and $\mathrm{S} 2$, the peak in applied stress is reached at incipient cracking.

Photographs of three different bending specimens $(b=3 \mathrm{~mm})$ tested by Tagarielli et al. [15] are illustrated in Fig. 6, showing that fracture was not initiated at mid-span in the experiments (a marker line denotes the position of the loading roller in the experiments), as predicted by the MCS, compare Fig. 5a.

Deformed beam configurations obtained for two realisations of RF4 (denoted as S3 and S4) are shown in Fig. 7a for $b=3 \mathrm{~mm}$ and $G_{f}=300 \mathrm{Nm}^{-1}$. Similar to what shown in Fig. 5a for the case of RF1, cracks were not initiated at mid-span. Because RF4 allows variations in material strength across the $y-z$ plane (see Fig. 3a), asymmetric failure mechanisms are observed in this case, as evident in Fig. 7b. Cracks initiated at the bottom of the beam (left) or at the vertical lateral surfaces (right) depending on the location of the weakest SVE in each realisation, and on the stress field induced. Figure 7c presents the corresponding stress versus displacement curves for both realisations S3 and S4. It is interesting to note that crack propagation was initially stable, thanks to material points with high strength surrounding the crack front and acting as 'crack stoppers', and became unstable after the crack front had slowly grown to a sufficient size, represented by contour 2 in the case of Fig. 7b.

In Fig. 8a, the predicted stress versus mid-span displacement curves are plotted for five realisations of RF1, while those obtained for five RF4 realisations are presented in Fig. 8b. In both these figures, the empty circles located on the curves indicate the crack initiation point; 
measurements by Tagarielli et al. [15] are included for comparison and are represented by the immediately after the crack is initiated, recall Fig. 5b. In contrast, if RF4 is employed, catastrophic fracture is delayed; a statistical toughening effect manifests, which brings predictions in good agreement with the measurements. We also include in Figs. 8a and 8b the predictions obtained from the reference simulations where the fracture stress was set equal to the measured average strength in uniaxial tension, $\sigma_{\mathrm{f} 0}=70 \mathrm{MPa}$; these predictions substantially under-predict the experimental results.

In order to explore the sensitivity of the apparent strength to the choice of random field, additional MCS were conducted on beams with RF2, RF3 and RF5, all of which of width $b=3 \mathrm{~mm}$ and fracture energy $G_{f}=300 \mathrm{Nm}^{-1}$, as illustrated in Fig. 3b. The finite element and statistical mesh sizes used in these simulations are listed in Table 1. In Fig. 9a, we present the corresponding MCS predictions of the average crack initiation stress and failure stress versus the normalised SVE size, defined as

$$
\bar{A}_{S V E}=\frac{A_{S V E}}{A}
$$

where $A_{S V E}$ denotes the area of interfaces contained in a single SVE and $A$ is the total crosssection of the beam, $A=b h$; numerical values of $\bar{A}_{S V E}$ are included in Table 1 . The band in Fig. 9a represents the range of the measured scatter in strength for beams of width $b=3 \mathrm{~mm}$. We also include error bars for the obtained strength predictions in Fig. 9a, indicating one standard deviation on either side of the mean. It can be seen from Fig. 9a, that in the MCS with $\operatorname{RF} 1\left(\bar{A}_{S V E}=1\right)$ and $\operatorname{RF} 2\left(\bar{A}_{S V E}=0.1\right)$, the beams failed catastrophically (i.e. failure stress and crack initiation stress coincide) and the corresponding predictions are well below the measurements. As the statistical mesh is increasingly refined (i.e., values of $\bar{A}_{S V E}$ are decreased) the statistical toughening effect is activated and the predicted beam strength progressively increases, while the crack initiation stress only shows a mild increase. This reduces the discrepancy between predictions and measurements, with RF3 showing the best correlation in this case.

To examine the effect of fracture energy $G_{\mathrm{f}}$ on the predicted strength, further sets of MCS were performed with RF1, for a range $300 \mathrm{Nm}^{-1} \leq G_{f} \leq 12 \mathrm{kNm}^{-1}$ on beams of width $b=3 \mathrm{~mm}$, see Table 1. The obtained predictions of failure and crack initiation stress are plotted in Fig. 
$9 \mathrm{~b}$ as functions of $G_{\mathrm{f}}$; again, error bars (equal to one standard deviation) and experimental

\subsubsection{Size dependence of the predicted strength}

Having examined the sensitivity of the predictions to the choice of random field, we now quantify the predicted size effect.

In order to examine the sensitivity of the apparent strength upon the sample size, additional sets of MCS were conducted with RF1 and RF5 on beams of width $b=15 \mathrm{~mm}$ and $b=53 \mathrm{~mm}$, using a fracture energy of $G_{f}=300 \mathrm{Nm}^{-1}$. The random fields were scaled to larger sizes by keeping their respective $\bar{A}_{S V E}$ parameter constant (see Table 1); in doing so, the length scale across which the material strength varies scales in proportion with the beam size.

In Fig. 10a we present the average failure stresses of the MCS response population (sample size $N_{\text {sim }}=10$ ) as functions of the beam volume for both RF1 and RF5, together with the results obtained from the deterministic reference simulations (dashed curve). Analytical predictions obtained from our modified Weibull model (eq. (9)) are included for the choice $\sigma_{0}=75 \mathrm{MPa}, m=13$ and $A_{0}=430 \mathrm{~mm}^{2}$, in line with the measurements; these predictions 
are indicated by the grey band in Fig. 10a, where the upper and lower bounds represent realisations, $\sigma_{\mathrm{f}}$ should lie within this band). Predictions of eq. (9) are shown as a reference only; the Weibull model cannot capture the ultimate strength of the beams, however it is expected to predict the load at initiation of failure; the predictions reported in Figs. 9a and 10a confirm that this is the case.

It is clear from Fig. 10a that for both cases RF1 and RF5, the MCS capture the Weibull-type size effect on the nominal strength, as predicted by eq. (9). This size effect was invoked by the implemented scaling of material strength with size of the SVEs, according to eq. (6), and by maintaining the spatial variability of strength $\left(\bar{A}_{S V E}=\right.$ const. $)$. On the other hand, the failure stresses obtained from the deterministic FE simulations (with uniform strength) are found to be insensitive to the specimen size, concluding that the size effects predicted by the MCS are of purely statistical nature. We also note that the MCS predictions performed with RF5 (with the same $G_{f}=300 \mathrm{Nm}^{-1}$ ) show a significant strengthening compared to the RF1 case, consequent to the statistical effect discussed in Section 4.2.1 and illustrated in Fig. 9a.

In Fig. 10b we compare the MCS predictions presented in Fig. 10a to the measurements by Tagarielli et al. (2010). In order to illustrate the scatter of the MCS response populations, we include in Fig. 10b error bars, indicating one standard deviation on either side of the mean. It can be seen that most of the measurements lie within these two curves. The scatter in the experiments is due to inevitable manufacturing imperfections present at different lengthscales in this thick laminate, in addition to the imperfections at the interfaces that this study attempts modelling. The predictions obtained from the MCS with RF5 and RF1 appear to provide effective upper and lower bounds for the apparent strength of the interface, respectively. The fact that RF1 appears as a lower bound is logical because it replicates the most brittle random strength configuration (no variation of strength across the fracture plane, see Fig. 3). Successive refinement of the statistical mesh (i.e. decreasing the SVE size in the RF) activates more complex failure modes and results in statistical toughening and higher apparent strength. 


\section{CONCLUSIONS}

FE simulations were performed in order to predict the interlaminar failure of a carbon/epoxy laminate subject to bending, and to compare to previously published measurements. The Weibull distribution of the interlaminar tensile strength measurements was used as an input for the numerical models. Discrete random fields of failure properties were used in the FE simulations to model the spatial variability of material strength, and a Monte Carlo analysis was performed. In addition, a modified Weibull scaling theory was developed using classical beam theory and considering failure along interfaces. We explored the sensitivity of the predictions to the type of random field used and to the value of material toughness. The FE predictions of failure modes and tensile strength were found in good agreement with the experimental observations and measurements. The main conclusions of this study are:

- It was shown that a deterministic modelling approach underestimates the structural strength and cannot capture the complex failure modes induced by material strength variability.

- Implementing random fields of material strength allows capturing asymmetric failure modes as those observed in the experiments.

- Increasing the statistical mesh density (or equivalently, decreasing the material lengthscale) leads to an increase in ultimate beam strength, independent of the choice of fracture energy. This is due to the more complex crack front shapes and paths induced by the variability in strength, resulting in a statistical toughening effect.

- The amount of statistical toughening was found to be dictated by the non-dimensional parameter $\bar{A}_{S V E}$. The lowest strength was predicted for $\bar{A}_{S V E}=1$, corresponding to the case of beams with no random variation of strength across the fracture plane.

- It was shown that using the fracture toughness as a calibration parameter for the material response leads to incorrect predictions of the deformation mechanisms and of the ultime failure load.

- For beams of constant non-dimensional material length scale $\bar{A}_{S V E}$, the MCS adequately predict the measured dependence of beam strength upon sample size.

- For an appropriate choice of random field, the proposed approach could predict the beam strength and its dependence upon volume, at identical computational cost to that of the deterministic simulations. 


\section{Acknowledgements}

The authors are grateful to EPSRC for funding through the Doctoral Prize Fellowship granted to Dr Schiffer at Imperial College London, Department of Aeronautics. 


\section{References}

[1] Wisnom MR. Size effects in the testing of fibre-composite materials. Compos Sci Technol. 1999;59:1937-1957.

[2] Weibull W. A statistical distribution function of wide applicability. J Appl Mech. 1951;18:293-297.

[3] Jackson KE, Kellas S. Effect of specimen size on the tensile and flexural strength of geometrically scaled composite laminates. US Army Symposium on Solid Mechanics, Plymouth MA1993.

[4] Jackson KE. Scaling effects in the flexural response and failure of composite beams. AIAA Journal. 1992;30:2099-2105.

[5] Wisnom MR. The effect of specimen size on flexural strenth of unidirectional carbon fibre-epoxy. Compos Struct. 1991;18:47-63.

[6] Rosen BW. Tensile Failure of Fibrous Composites. AIAA Journal. 1964;2:1985-1991.

[7] Wisnom MR. Relationship between strength variability and size effect in unidirectional carbon fibre/epoxy. Composites. 1991;22:47-52.

[8] Gurvich MR, Pipes RB. Strength size effect of laminated composites. Compos Sci Technol. 1995;55:93-105.

[9] Okabe T, Takeda N. Size effect on tensile strength of unidirectional CFRP composites experiment and simulation. Compos Sci Technol. 2002;62:2053-2064.

[10] Curtin WA, Takeda N. Tensile Strength of Fiber-Reinforced Composites: I. Model and Effects of Local Fiber Geometry. J Compos Mater. 1998;32:2042-2059.

[11] Bazant ZP, Daniel IM, Li Z. Size Effect and Fracture Characteristics of Composite Laminates. Journal of Engineering Materials and Technology. 1996;118:317-324.

[12] Bazant ZP. Scaling Laws in Mechanics of Failure. Journal of Engineering Mechanics. 1993;119:1828-1844.

[13] O'Brian TK, Salpekar SA. Scale effects on the transverse tensile strength of graphite epoxy composites. In: E. C, editor. Composite Materials: testing and design, vol. 111995.

[14] Wisnom MR, Jones MI. Size effects in interlaminar tensile and shear strength of unidirectional glass fibre-epoxy. Journal of Reinforced Plastics and Composites. 1996;15:2-15.

[15] Tagarielli VL, Minisgallo G, McMillan AJ, Petrinic N. The response of a multidirectional composite laminate to through-thickness loading. Compos Sci Technol. 2010;70:1950-1957.

[16] Hill R. Elastic properties of reinforced solids: some theoretical principles. J Mech Phys Solids. 1963;11:357-372.

[17] Drugan WJ, Willis JR. A micromechanics-based nonlocal constitutive equation and estimates of representative volume element size for elastic composites. J Mech Phys Solids. 1996;44:497-524.

[18] Kanit T, Forest S, Galliet I, Mounoury V, Jeulin D. Determination of the size of the representative volume element for random composites: statistical and numerical approach. Int J Solids Struct. 2003;40:3647-3679.

[19] Ostoja-Starzewski M. Material spatial randomness: From statistical to representative volume element. Probabilistic Engineering Mechanics. 2006;21:112-132. 
[20] Trias D, Costa J, Turon A, Hurtado JE. Determination of the critical size of a statistical representative volume element (SRVE) for carbon reinforced polymers. Acta Materialia. 2006;54:3471-3484.

[21] Bazant ZP, Pang SD, Vorechovsky M, Novak D, Pukl R. Statistical size effect in quasibrittle materials: Computation and extreme value theory. In: al. Le, editor. Int Conf on Fracture Mech of Concrete and Concr Structures, 2004.

[22] Yin X, Chen W, To A, McVeigh C, Liu WK. Statistical volume element method for predicting microstructure-constitutive property relations. Computational methods in applied mechanics and engineering. 2008;197:3516-3529.

[23] Stefanou G. The stochastic finite element method: Past, present and future. Computer Methods in Applied Mechanics and Engineering. 2009;198:1031-1051.

[24] Song JH, Areias PMA, Belytschko T. A Method for Dynamic Crack and Shear Band Propagation with Phantom Nodes. International Journal of Numerical Methods in Engineering. 2006;67(868-893). 
(a)

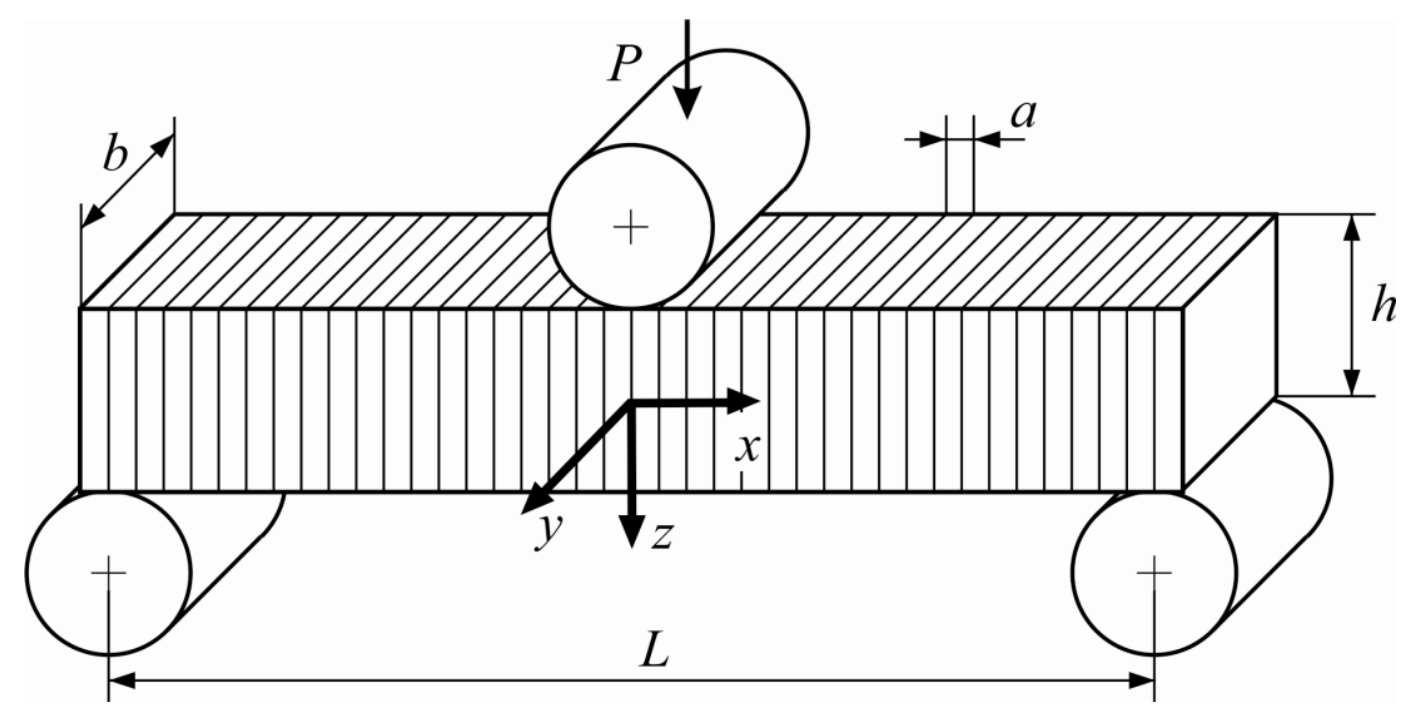

(b)

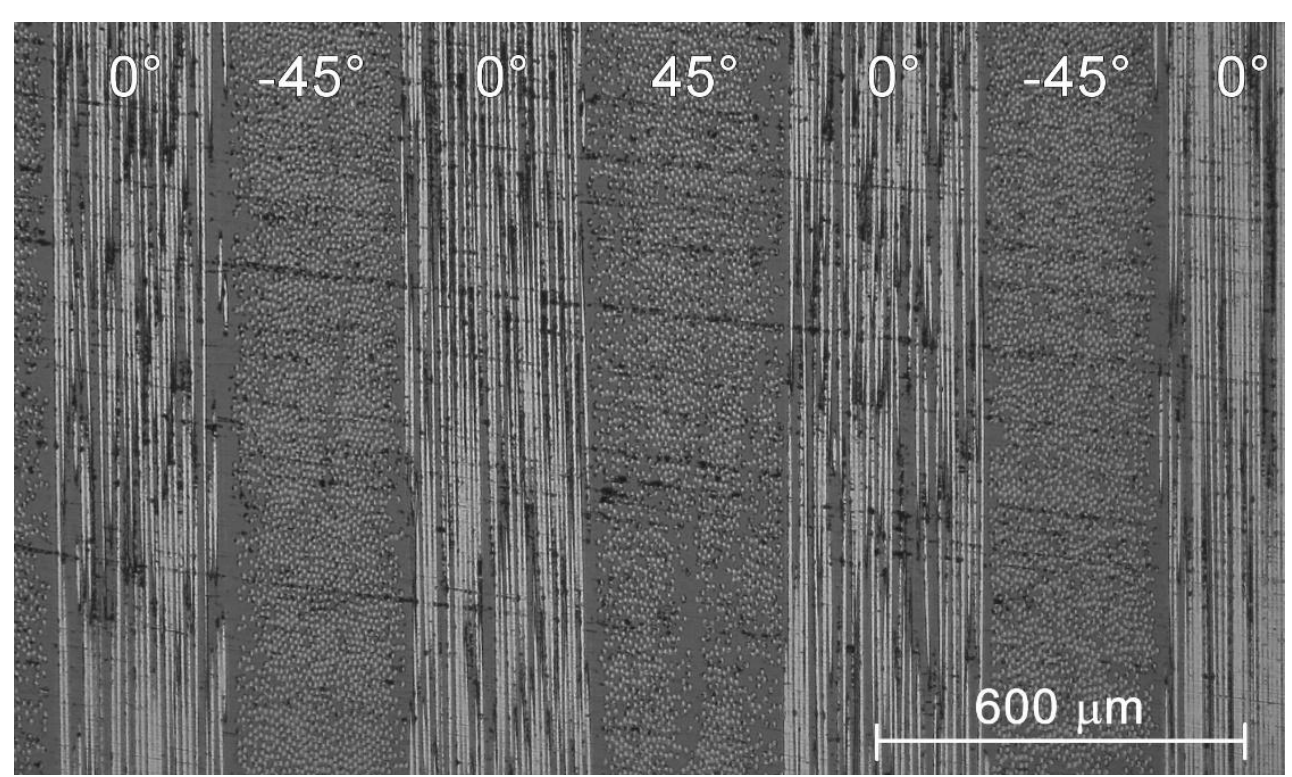

Fig. 1 (a) Sketch of the problem geometry, showing the bending loading on the multi-directional composite laminate; (b) Micrograph showing the microstructure of the composite laminate. 

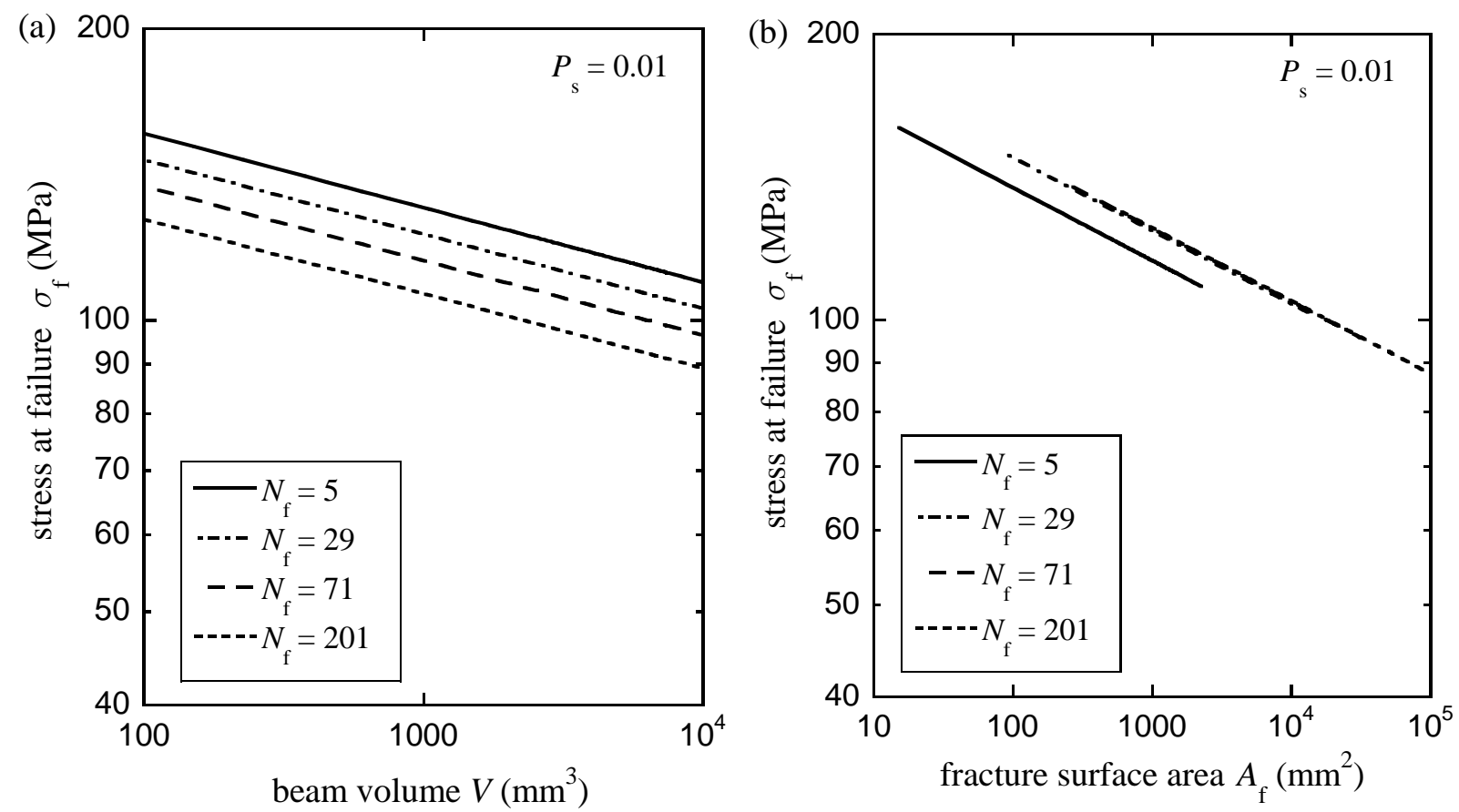

Fig. 2 Theoretical predictions (eq. (9)) of failure stress versus (a) beam volume and (b) fracture surface area, for beams in three-point bending obtained using $P_{s}=0.01, \sigma_{0}=75 \mathrm{MPa}, m=13$ and $A_{0}=430 \mathrm{~mm}^{2}$; predictions for four different numbers of interfaces $N_{\mathrm{f}}$ are shown. 

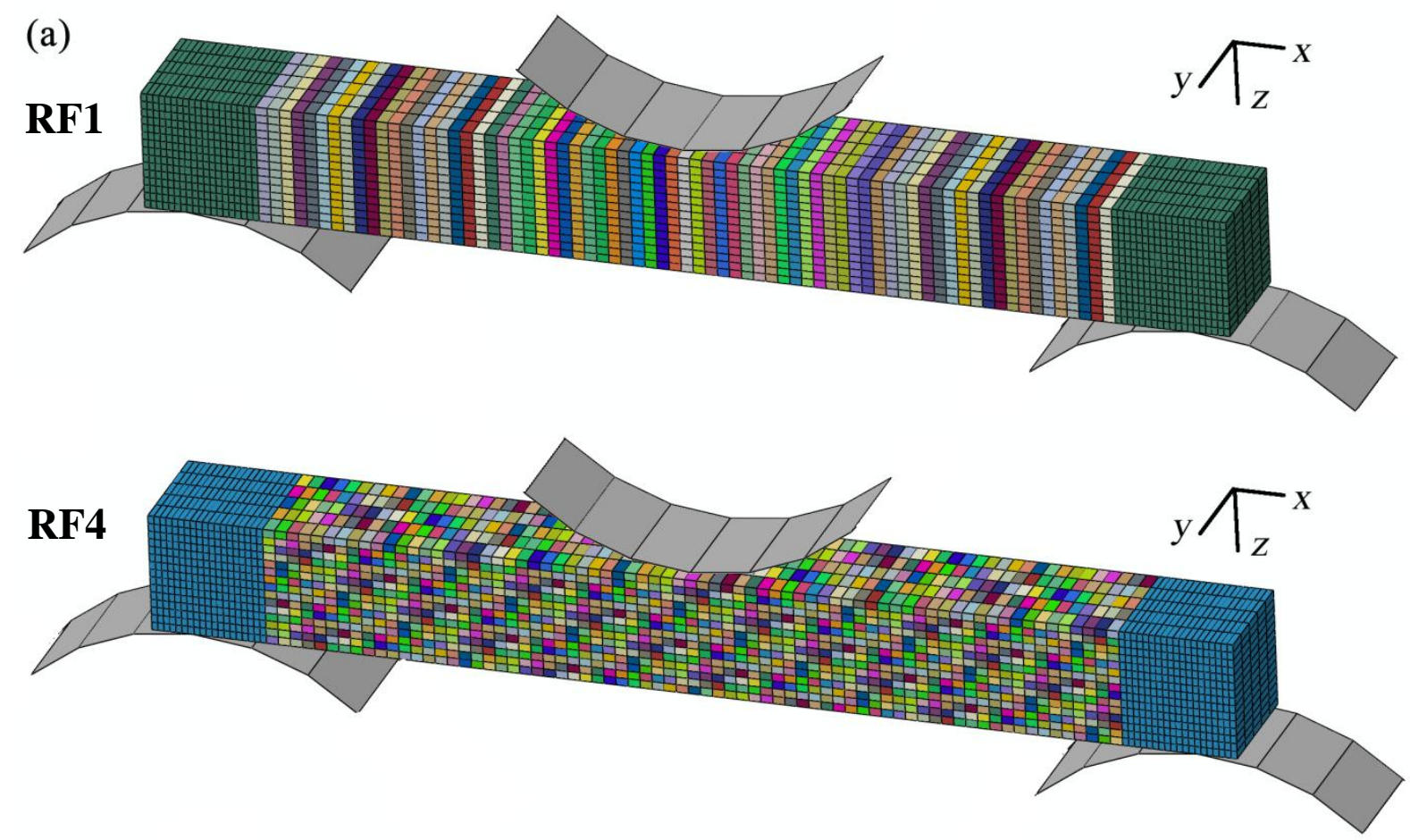

(b)

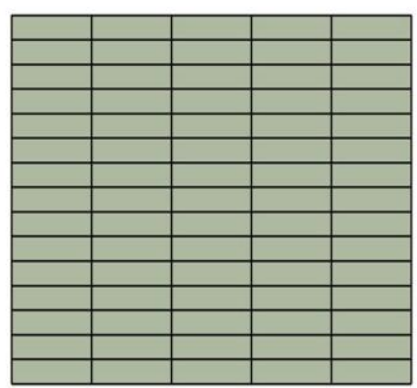

RF1

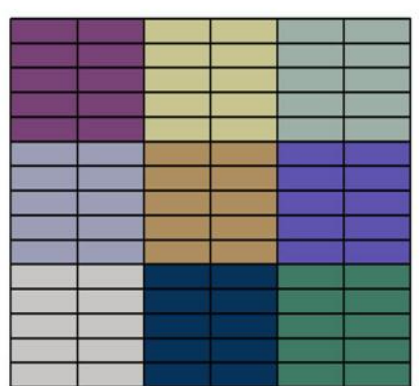

RF2

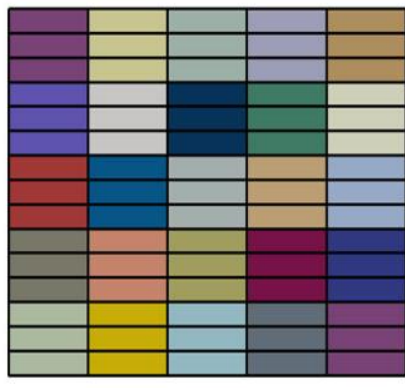

RF3

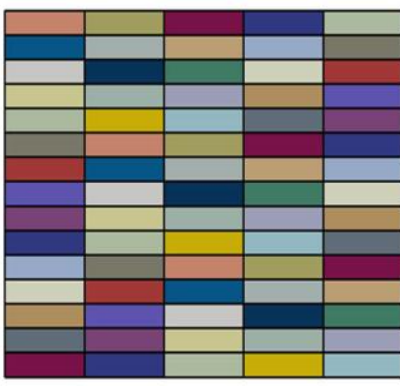

RF4

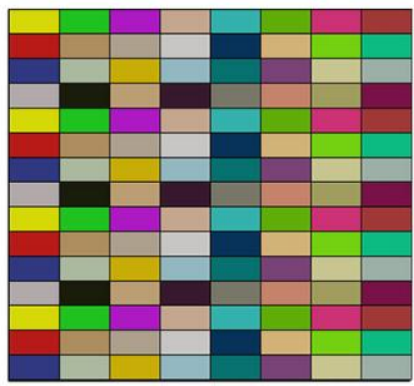

RF5

Fig. 3 (a) Illustration of the FE model used to perform MCS on a beam of width $b=3 \mathrm{~mm}$ with RF1 (top) and RF4 (bottom) representations of material strength variability; the coloured mesh represents the statistical discretisation and the FE mesh is shown as a black wireframe; (b) cross-sections through the $\mathrm{y}-\mathrm{z}$ plane of beams of width $b=3 \mathrm{~mm}$, showing the various RF representations of random strength considered in this study. 
(a)

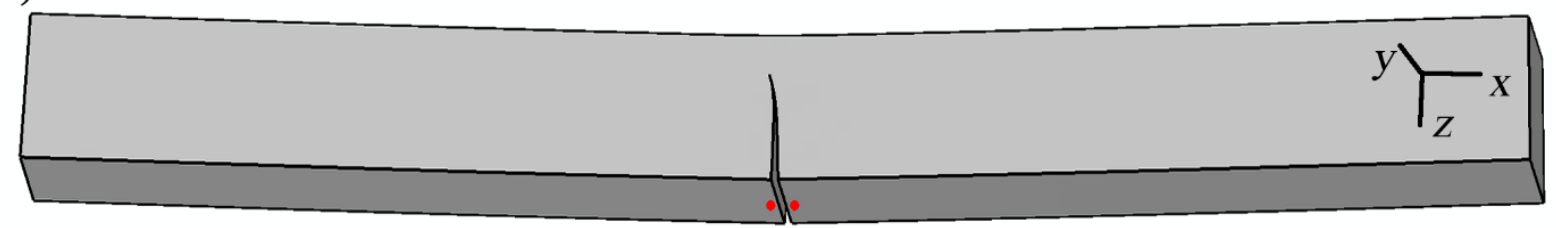

(b)
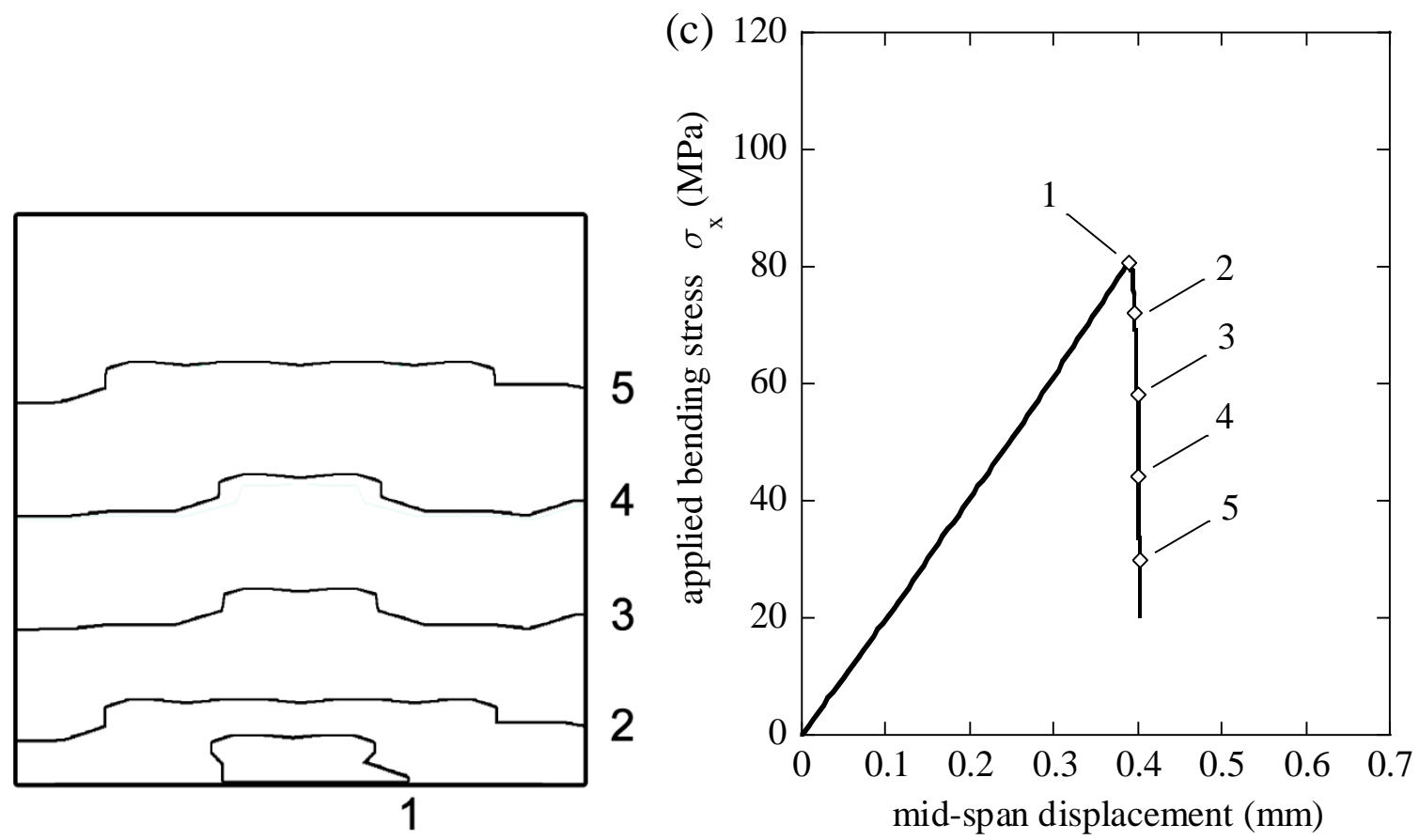

Fig. 4 Failed configuration (a), evolution of crack front (b) and applied bending stress as a function of mid-span displacement (c) obtained from a deterministic FE simulation for a beam of width $b=15 \mathrm{~mm}$, uniform failure initiation stress $\sigma_{\mathrm{f} 0}=70 \mathrm{MPa}$ and fracture energy $G_{f}=300 \mathrm{Nm}^{-1}$. 
(a)

S1

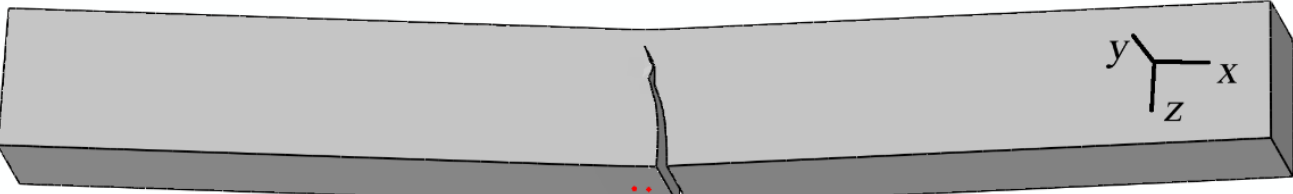

S2

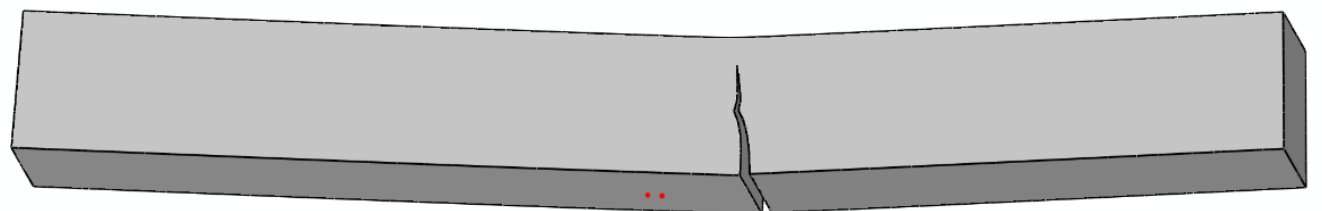

(b)
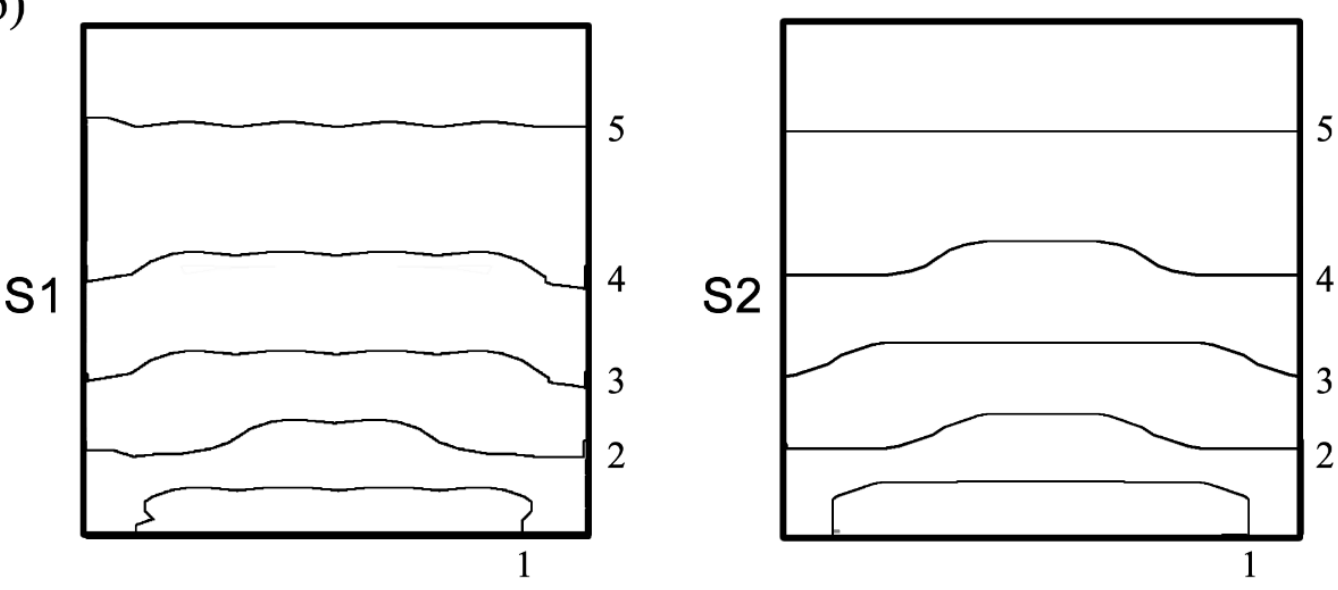

(c)
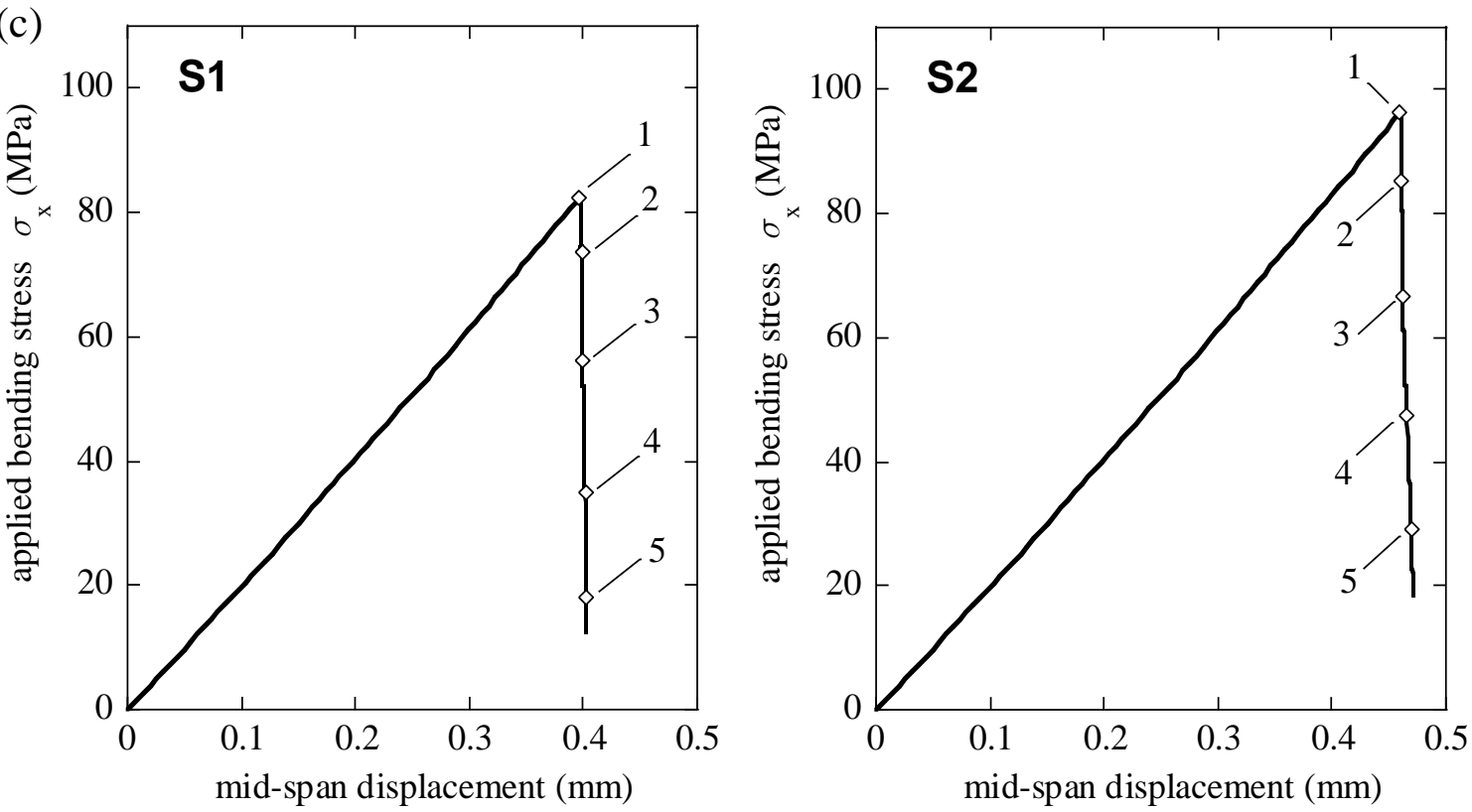

Fig. 5 Finite Element predictions of failed configuration (a) and crack front evolution (b) for two realisations of RF 1, denoted here as S1 and S2, respectively; results are presented for a beam of width $b=3 \mathrm{~mm}$ and fracture toughness $G_{f}=300 \mathrm{Nm}^{-1}$; (c) applied bending stress as a function of mid-span displacement for both RF1 realisations. 

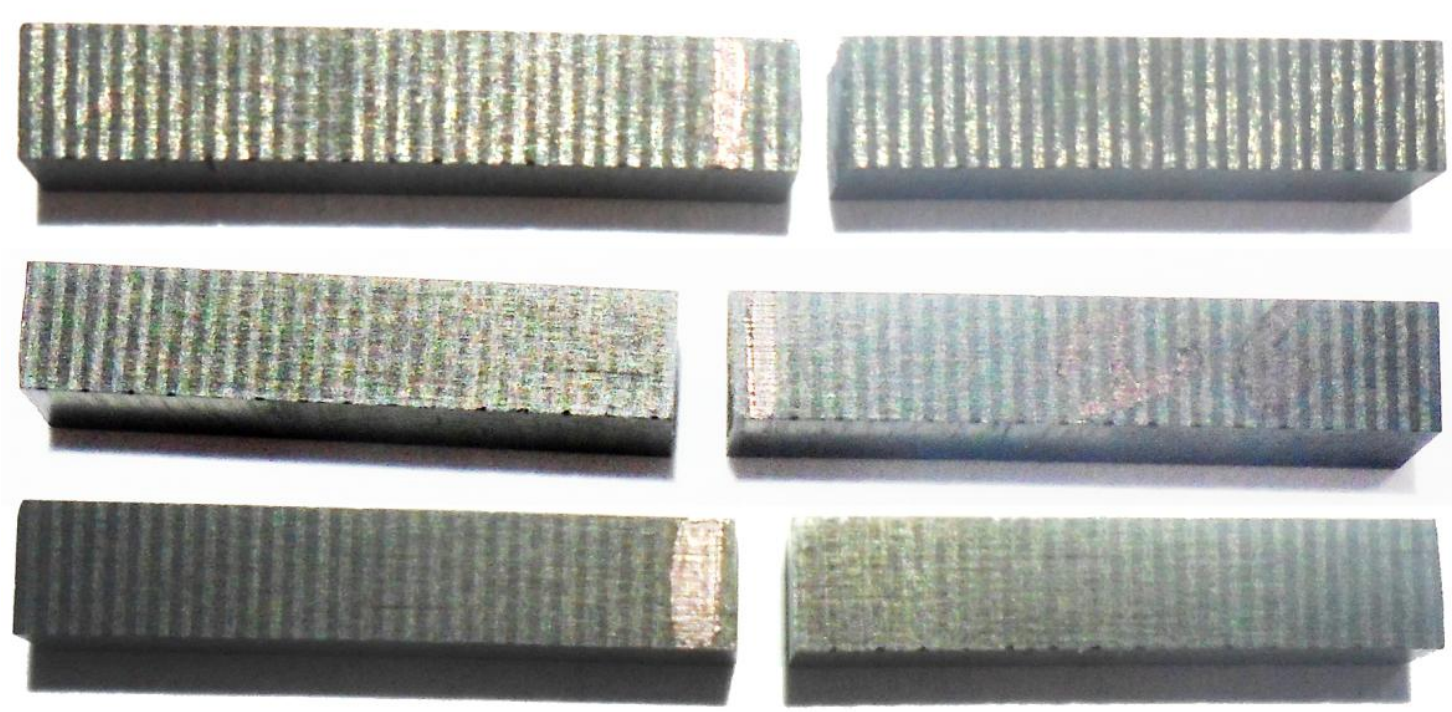

Fig. 6 Post-test photographs of the tested composite beams, showing evidence of asymmetric failure mechanisms. 
(a)

S3

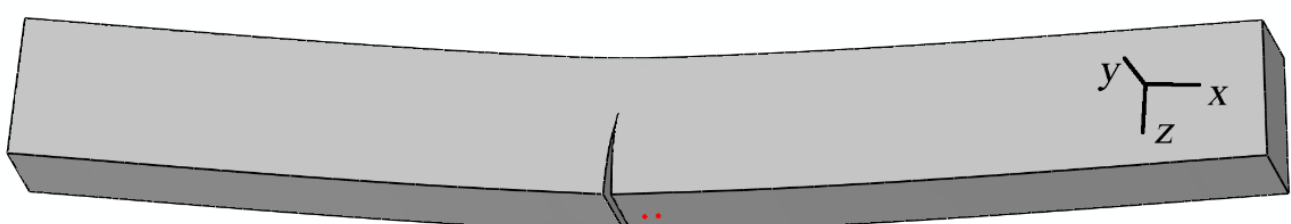

S4

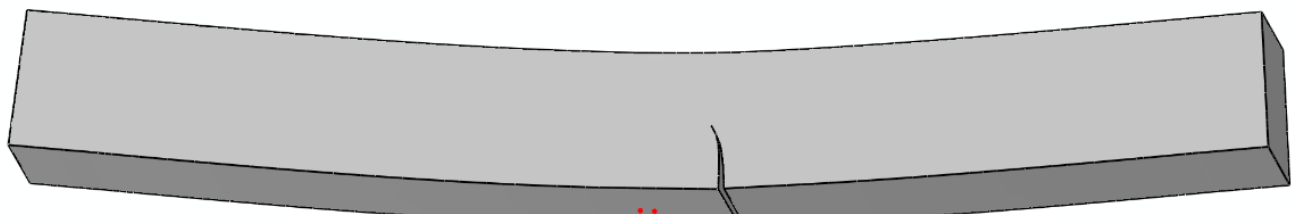

(b)
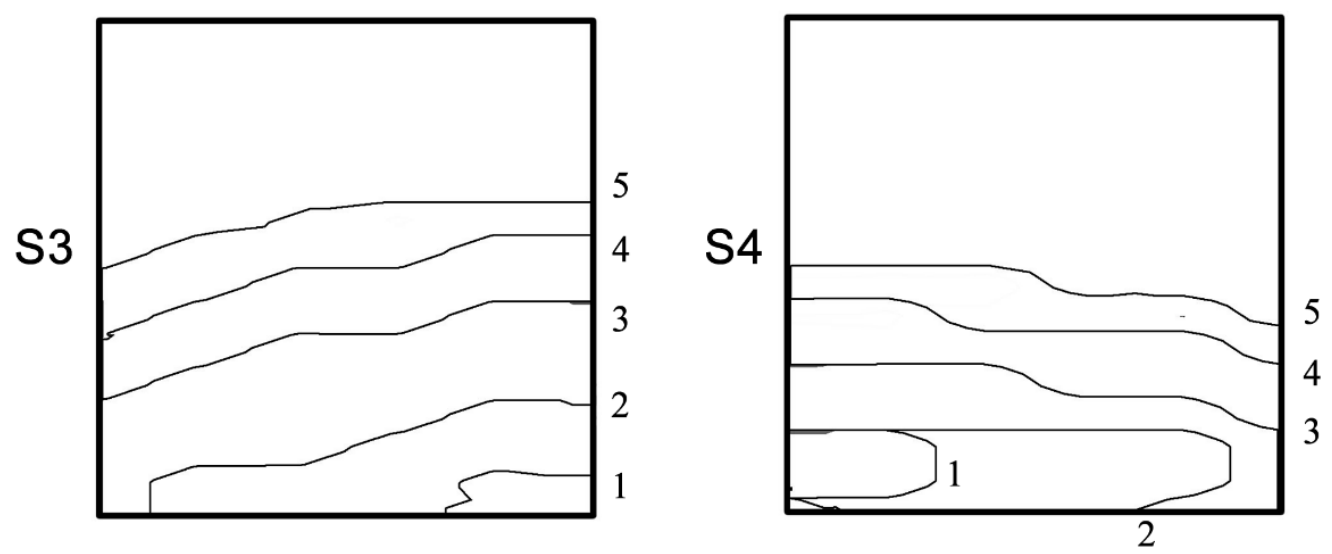

(c) $2 0 0 \longdiv { S 3 }$
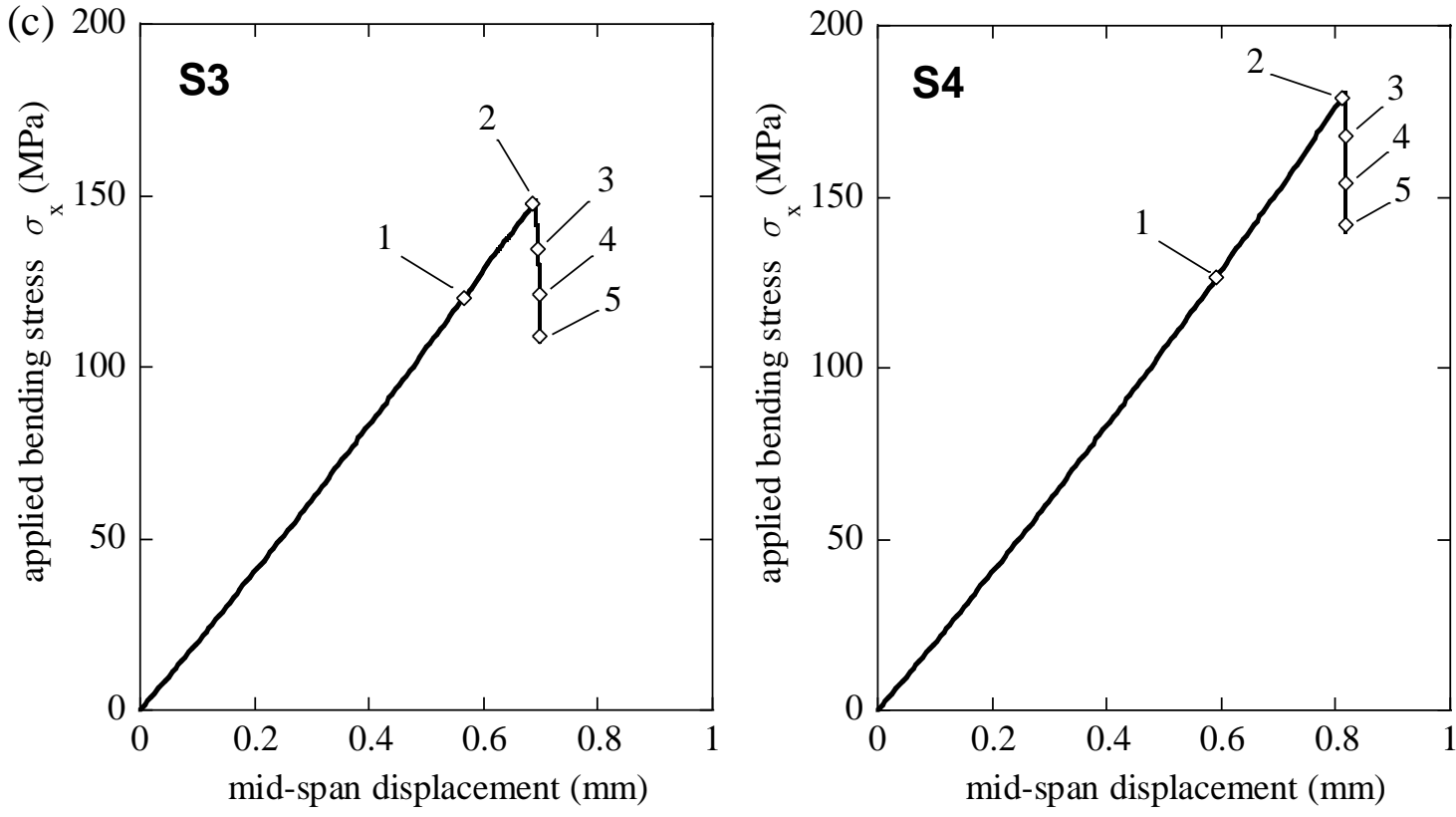

Fig. 7 Finite Element predictions of failed configuration (a) and crack front evolution (b) for two realisations of RF 4, denoted here as S3 and S4, respectively; results are presented for a beam of width $b=3 \mathrm{~mm}$ and fracture toughness $G_{f}=300 \mathrm{Nm}^{-1}$; (c) applied bending stress as a function of mid-span displacement for both RF4 realisations. 

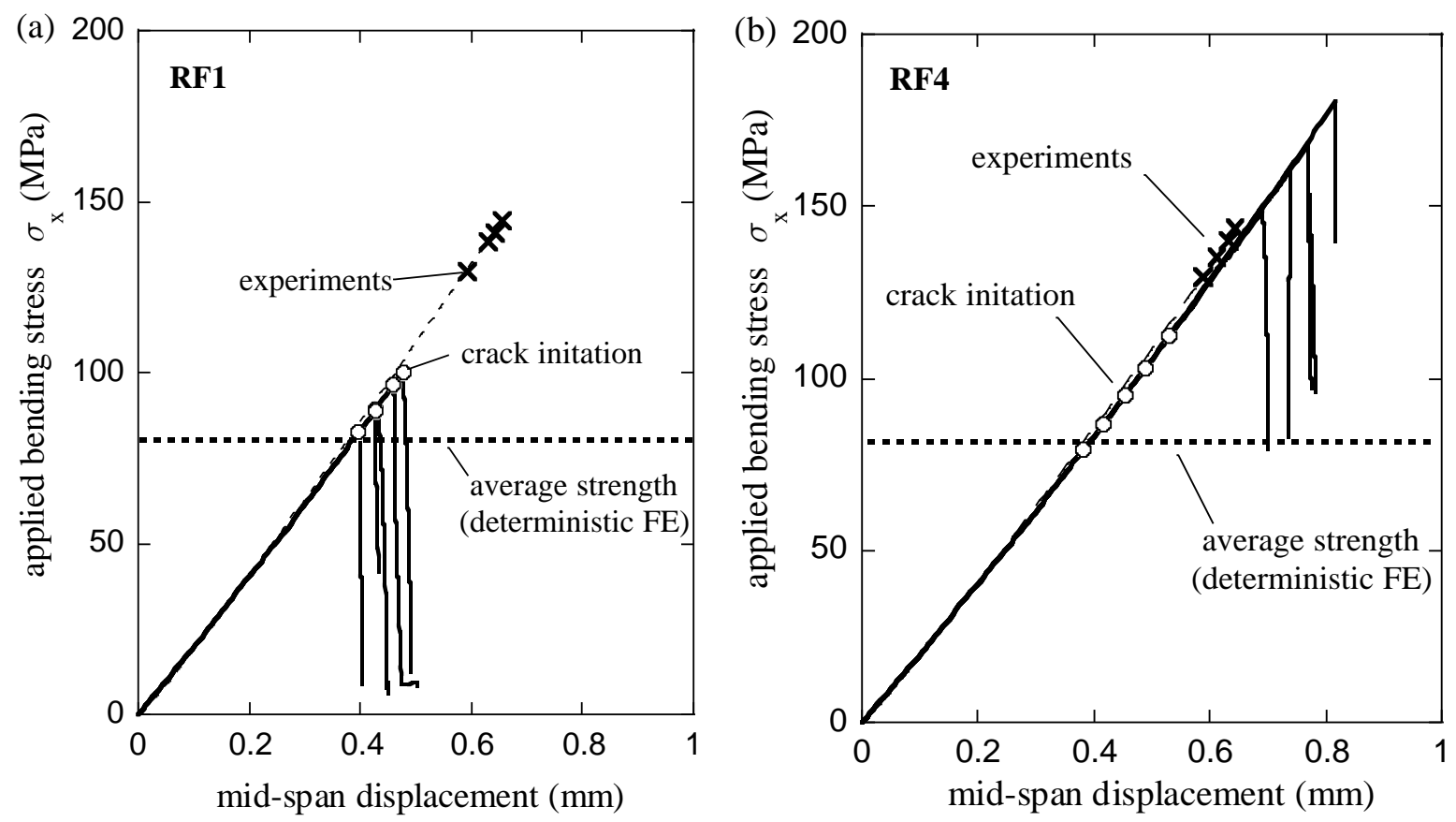

Fig. 8 (a) Applied bending stress versus displacement curves obtained from 5 realisations of RF 1, for $b=3 \mathrm{~mm}$ and $G_{f}=300 \mathrm{Nm}^{-1}$; (b) similar information for the case of RF 4 . The empty circles denote the onset of failure; measurements by Tagarielli et al. (2010) are included for comparison. The horizontal lines indicate the ultimate failure stress predictions of the deterministic reference simulation. 

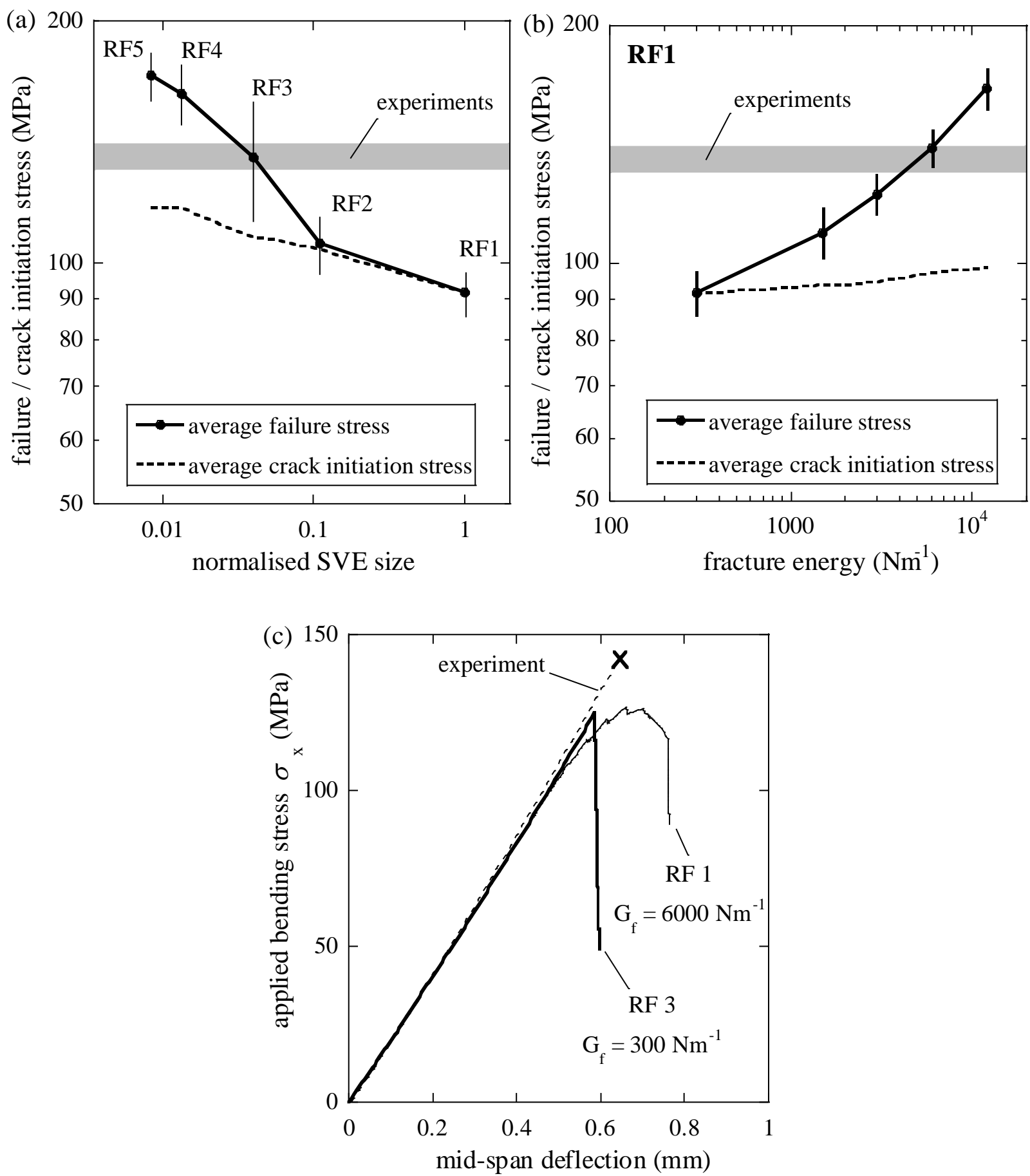

Fig. 9 (a) Average crack initiation and ultimate failure stresses obtained from the MCS simulations for various random fields ( $b=3 \mathrm{~mm}$ and $G_{\mathrm{f}}=300 \mathrm{Nm}^{-1}$ ); the band represents the measurements by Tagarielli et al. (2010); the error bars shown represent one standard deviation on either side of the mean; (b) average failure and crack initiation stresses as functions of the fracture energy, $G_{\mathrm{f}}$, for the case of RF1 ( $b=3 \mathrm{~mm})$; (c) applied bending stress as a function of the mid-span deflection for the cases RF1 $\left(G_{\mathrm{f}}=\right.$ $\left.6000 \mathrm{Nm}^{-1}\right)$ and $\operatorname{RF} 3\left(G_{\mathrm{f}}=300 \mathrm{Nm}^{-1}\right)$, for $b=3 \mathrm{~mm}$; measurements are included for comparison. 

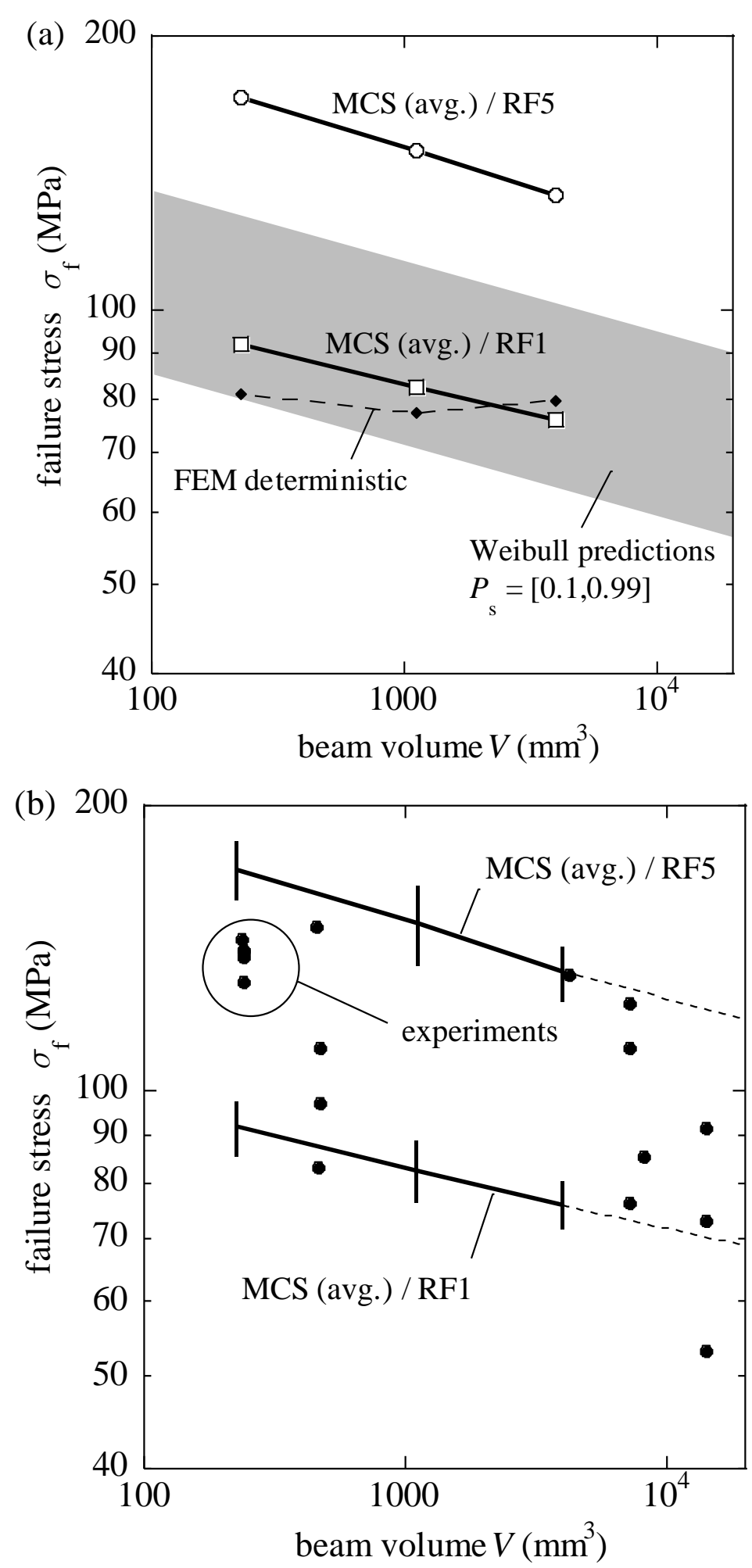

Fig. 10 (a) Average failure stresses obtained from the MCS response population (sample size $N_{\text {sim }}=10$ ) as functions of the beam volume for $G_{f}=300 \mathrm{Nm}^{-1}$; results obtained for RF 1 and RF5 are included and compared to the deterministic FE results; the band represents analytical (Weibull) predictions obtained from eq. (9); (b) comparison between the measurements by Tagarielli et al. (2010) and the MCS results presented in (a); the error bars represent one standard deviation on either side of the mean value; the dotted lines indicate the extrapolated portion of the MCS curves. 
Table 1: Finite element and statistical mesh sizes for the sets of MCS performed in this study.

\begin{tabular}{cccccccccc}
\hline \multirow{2}{*}{$\begin{array}{c}\text { width } b \\
(\mathrm{~mm})\end{array}$} & RF & \multicolumn{2}{c}{ Finite element size $(\mathrm{mm})$} & \multicolumn{2}{c}{ Statistical mesh size (mm) } & FEs per & $\bar{A}_{S V E}$ \\
\hline 3 & & $l_{\mathrm{x}}$ & $l_{\mathrm{y}}$ & $l_{\mathrm{z}}$ & $l_{\mathrm{x}}$ & $l_{\mathrm{y}}$ & $l_{\mathrm{z}}$ & SVE & \\
\hline 3 & RF2 & 0.3 & 0.3 & 0.6 & 0.3 & 3 & 3 & 75 & 1 \\
\hline 3 & RF3 & 0.3 & 0.2 & 0.6 & 0.3 & 1 & 1 & 10 & 0.11 \\
\hline 3 & RF4 & 0.3 & 0.2 & 0.6 & 0.3 & 0.2 & 0.6 & 1 & 0.013 \\
\hline 3 & RF5 & 0.3 & 0.2 & 0.375 & 0.3 & 0.2 & 0.375 & 1 & 0.008 \\
\hline 15 & RF1 & 0.3 & 0.2 & 0.6 & 0.3 & 3 & 15 & 375 & 1 \\
\hline 15 & RF5 & 0.3 & 0.2 & 0.6 & 0.3 & 0.6 & 0.6 & 3 & 0.008 \\
\hline 53 & RF1 & 0.3 & 0.2 & 2.12 & 0.3 & 3 & 53 & 375 & 1 \\
\hline 53 & RF5 & 0.3 & 0.2 & 2.12 & 0.3 & 0.6 & 2.12 & 3 & 0.008 \\
\hline
\end{tabular}

